\title{
Magnetic Resonance Imaging in Hematospermia: Does It Increase Unnecessary Prostate Biopsy?
}

\author{
Luke A. McGuinness ${ }^{\mathrm{a}} \quad$ Samer Obeidat $^{\mathrm{a}} \quad$ Christopher Powell $^{\mathrm{b}}$ \\ a Department of Urology, Royal Liverpool and Broadgreen Hospitals, Liverpool; ${ }^{b}$ Department of Urology, \\ Countess of Chester Hospital, Chester, UK
}

\section{Key Words}

Hematospermia • Prostate cancer •

Magnetic resonance imaging

\begin{abstract}
Introduction: Hematospermia is an uncommon presentation of prostate cancer. Following the introduction of MRI for patients with hematospermia we evaluated its use and effect on prostate biopsy and cancer detection rates. Materials and Methods: Analysis of patients attending our outpatient department over 2 years was undertaken. Diagnostic workup included digital rectal examination and PSA. Those with abnormal findings or persisting symptoms were offered prostate biopsy. In the second year MRI became available for patients with hematospermia. Abnormal MRI or persisting symptoms were offered biopsy. We compared the frequency of prostate biopsy and cancer detection in patients undergoing MRI and those not having imaging. Results: Forty-seven patients were referred with hematospermia. Nineteen patients did not undergo MRI; four received prostate biopsy with one adenocarcinoma found. Twenty-four patients had an MRI with 17 biopsies undertaken. Three biopsies revealed adenocarcinoma. In the MRI group $71 \%$ of patients underwent prostate biopsy but only $21 \%$ from the non-MRI group $(p<0.05)$. Prostate cancer detection rate in the MRI group was $18 \%$ whilst in the non-MRI group was $25 \%(p=0.7)$. Con-
\end{abstract}

\section{KARGER}

(C) 2016 S. Karger AG, Basel

Fax +4161306 1234

E-Mail karger@karger.ch

www.karger.com
Accessible online at: www.karger.com/cur clusions: Our findings indicate that caution should be used with MRI as it can lead to an increase in prostate biopsy with no change in cancer detection rate.

Copyright $\odot 2016$ S. Karger AG, Basel

\section{Introduction}

Hematospermia or blood in the ejaculate is an uncommon presenting complaint but one that can cause understandable anxiety to the patient. The exact prevalence is unknown but it has been reported to account for 1 in 5,000 attendances to the urology clinic [1]. In most cases it is a benign, painless and self limiting condition requiring only simple investigations and reassurance [2]. Persistent or recurrent hematospermia warrants urological referral and investigation as there is an established association with prostate cancer [3,4]. There is no clear consensus on the imaging modalities that should be carried out in patients presenting with hematospermia; no set protocol exists but traditionally investigation involves transrectal ultrasound (TRUS) with or without prostate biopsy $[5,6]$. The role of magnetic resonance imaging (MRI) has not been fully established so far in the diagnostic workup for hematospermia but availability and ex- 


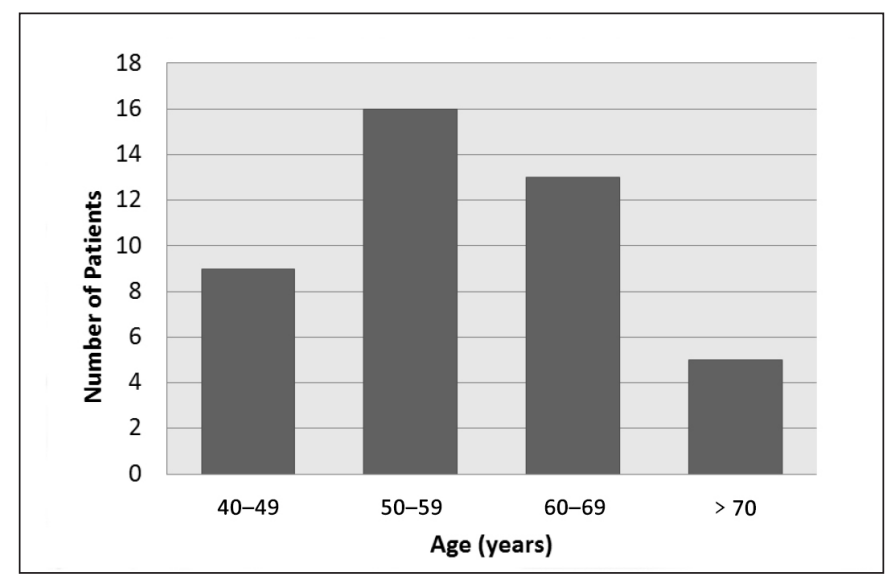

Fig. 1. Presenting age of hematospermia $(n=43)$.

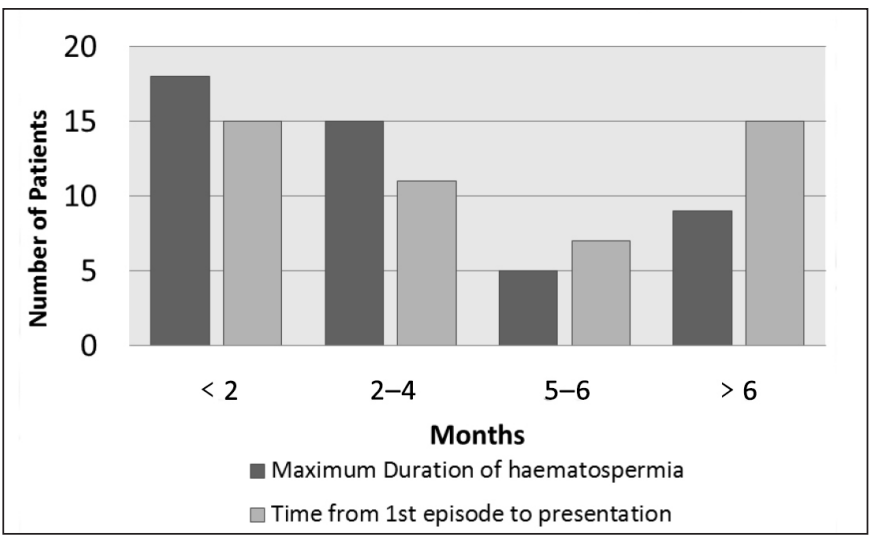

Fig. 2. Duration of hematospermia and time to presentation.

pertise in interpreting the images has steadily increased due to the use of MRI in prostate cancer staging. Thus the role of conventional and endorectal-MRI in investigating patients with hematospermia has led some to recommending its use as the evaluation modality of choice in hematospermia [7, 8]. There has been a recent trend, however, in the role of over investigation and treatment of clinically insignificant or low risk prostate cancer increasing evidence to support watchful waiting or active surveillance management $[9,10]$.

Prostate specific antigen (PSA) in combination with digital rectal examination (DRE) and MRI should provide extremely valuable, non-invasive, information in selecting patients requiring TRUS guided biopsies of the prostate.
Table 1. Patients not undergoing MRI $(n=19)$

\begin{tabular}{ll} 
Reason for not having MRI & Number \\
\hline Imaging modality unavailable & 16 \\
Abnormal DRE with subsequent biopsy & 2 \\
Pacemaker in situ & 1 \\
\hline
\end{tabular}

Table 2. Patients with abnormal MRI $(n=15)$

\begin{tabular}{ll}
\hline Abnormality on MRI & Number \\
\hline Reduced signal in peripheral zone (possible T2 lesion) & 10 \\
Seminal vesicle haemorrhage & 3 \\
Irregular peripheral zone & 2 \\
\hline
\end{tabular}

At our institution we incorporated the use of MRI into the investigative pathway for hematospermia. Our aim was to ascertain whether the introduction of MRI had led to a change in prostate biopsy and cancer detection rates in patients presenting with hematospermia.

\section{Materials and Methods}

We undertook a retrospective analysis of all 47 patients referred to our institution with recurrent or persistent hematospermia in a 2 year period from July 2009 -June 2011 inclusive. In 2010 MRI scan of the prostate was used for patients with hematospermia; we reviewed the outcomes in hematospermia patients one year before and after this. After 2011, due to the results of this study discussed later, MRI was removed from our hematospermia protocol. Prostate MRI was undertaken with a 1.5 Tesla Siemens Avanto MRI scanner. Standard MRI protocol using T1, T2 and Diffusion weighted images of the prostate gland to produce axial and coronal views with interpretation and reporting by a specialist uroradiologist. Patients were identified by our clinical coding department and their notes were assessed for information on duration of hematospermia, recurrent hematospermia, concurrent hematuria, anticoagulation use, history of urological malignancy, rectal examination, urine culture, PSA, MRI and TRUS guided prostate biopsy.

Patients $<40$ years old were considered low risk and were not offered MRI, PSA testing or prostate biopsy and therefore excluded from analysis. Patients $>40$ years with normal PSA and DRE were offered MRI where not contraindicated and subsequent TRUS guided prostate biopsy was performed depending on presence of imaging abnormality or patient wishes with appropriate counselling. 
Table 3. Summary of abnormal prostate biopsies $(n=5)$

\begin{tabular}{llll}
\hline Histology & DRE finding & PSA $(\mathrm{ng} / \mathrm{ml})$ & MRI Finding \\
\hline Gleason 6 adenocarcinoma & normal & 0.90 & reduced signal peripheral zone \\
Gleason 6 adenocarcinoma & normal & 1.02 & reduced signal peripheral zone \\
Gleason 7 (3+4) & normal & 1.25 & reduced signal peripheral zone \\
\hline
\end{tabular}

DRE $=$ Digital rectal examination.

Comparisons were made in the outcomes of patients undergoing MRI against those patients who presented before its introduction in our investigation pathway. Particular attention was paid to subsequent prostate biopsy and prostate cancer detection. Statistical significance was calculated using chi-squared testing.

\section{Results}

The median age at presentation was 57 years (fig. 1) with $91 \%$ (43/47) of patients being $>40$ years old being used in analysis. Most presented with haematospermia as the solitary complaint (37/43) but other associated symptoms included hematuria (3/43), infertility (1/43), erectile dysfunction (1/43), testicular pain (1/43) and reduced urinary flow (1/43). Thirty-nine $(91 \%)$ had recurrent episodes with an average duration of symptoms of 22 weeks at presentation (fig. 2). Urine culture indicated bacterial growth in 2 patients $(4.25 \%)$. Two patients had suspicious DRE and both patients underwent subsequent biopsy. Serum PSA testing was done on all patients; only one value was significant $(50.6 \mathrm{ng} / \mathrm{ml})$ in conjunction with a suspicious DRE whilst the remaining values had a mean of $0.95 \mathrm{ng} / \mathrm{ml}$ (range $0.21-2.51 \mathrm{ng} / \mathrm{ml}$; standard deviation 0.52).

Nineteen patients did not undergo an MRI (table 1) the majority presented prior to its introduction. Of this group four patients $(21 \%)$ went on to have prostate biopsy. One due to suspicious DRE had Gleason 9 adenocarcinoma and three with persistent hematospermia had benign histology.

Twenty-four patients went on to have an MRI scan; 15 patients were found to have an abnormality (table 2) and 9 patients were found to have no abnormality. All those with abnormal MRI subsequently had a prostate biopsy; 11 had benign histology and 3 revealed prostate adenocarcinoma (table 3). One patient had prostatic intraepithelial neoplasia on initial biopsy but benign on repeat biopsy. Two patients with a normal MRI had prostate biopsies for persistent hematospermia with appropriate patient counselling and both revealed benign histology.

Twenty-one patients (49\%) underwent a prostate biopsy; in the MRI group 17 patients (71\%) went on to have a prostate biopsy whilst in the non-MRI group 4 patients $(21 \%)$ had biopsy indicating a significant difference $(\mathrm{p}=0.0015)$. Of the 21 biopsies four were found to have prostate cancer (19\%) with an overall incidence of $8.5 \%$ in those presenting with hematospermia. Three patients from the MRI group receiving biopsy had cancer (18\%) whilst one patient in the non-MRI group undergoing biopsy had cancer (25\%) with no significant difference $(p=0.74)$.

\section{Limitations}

The sample size and low general incidence of prostate cancer in hematospermia with our retrospective analysis makes it difficult to draw any concrete conclusions, but we have found significant statistical significance in subsequent biopsy rates with no significant increase in cancer detection rates. The retrospective analysis also lends some selection bias to our sample in particular the heterogeneity that might exist between our 2 patient groups which would be avoided with a randomized double blinded study design. Furthermore 2 patients who had suspicious DRE had up front biopsy and MRI in these patients may have picked up an abnormality.

\section{Discussion}

The majority of cases of hematospermia with an identified cause are infective in origin which can be dealt with at the primary care level [11]. Recurrent or persistent he- 
matospermia warrants a urological referral with a multitude of benign causes including cysts, calculi, seminal vesiculitis and vascular malformations being implicated. However the urologist should be alert to the possibility of prostate cancer and should guide subsequent investigation.

In our institution $8.5 \%$ of patients presenting with haematospermia were identified as having prostate cancer; this is comparable with those reported in literature. A 2007 literature review of over 900 cases found prostate cancer in $3.5 \%$ [4] and a recent review of 300 cases reports $5.7 \%$ incidence [1]. A review of a prostate cancer screening program found that prostate cancer was present in $13.7 \%$ of those reporting hematospermia [3].

To our knowledge this is the first study examining prostate biopsy and cancer detection rates related specifically to MRI use in hematospermia. The development of multiparametric and diffusion-weighted MRI has increased its use as a diagnostic tool in detecting prostate cancer $[13,14]$. Although not widely documented the use of conventional MRI in diagnosis of hematospermia was first described in the late 1980s [15] and later Furuya et al. [16] showed good correlation of MRI at detecting seminal vesicle hemorrhage confirming with aspiration. The largest series using MRI in hematospermia was undertaken by Li et al. [17] who analysed over 100 patients with refractory hematospermia and confirmed MRI findings with seminal vesiculoscopy; they undertook TRUS guided prostate biopsy in $11 \%$ of patients $>40$ years old but no prostate cancer was detected. A further series by Prando [8] looked at 86 patients with hematospermia undergoing MRI with an endorectal coil. Like the series by Li et al. [17] the majority of abnormal findings were benign however $4 \%$ underwent TRUS prostate biopsy with $50 \%$ of these found to have prostate cancer. Other isolated case reports have also picked up rare prostate abnormalities such as amyloidosis or seminal vesicle melanoma on MRI of patients presenting with persistent hematospermia [18, 19]. A variety of literature review and clinical review articles have suggested that MRI with an endorectal coil should be the investigative modality of choice patients with hematospermia but only in those with refractory symptoms or with risk factors for prostate cancer [4, 20, 21].

As our experience shows MRI is useful in detecting abnormalities in the majority (62.5\%) of patients presenting with hematospermia. However we have also found a significant increase in the numbers of patients undergoing biopsy on the basis of MRI findings. Our detection rate of abnormality on biopsy was $19 \%$ (4/21) which is comparable to larger studies $[22,23]$ with $75 \%(3 / 4)$ having normal DRE and PSA with biopsy performed on the basis of MRI findings. It is also worth noting that $67 \%$ $(2 / 3)$ of the prostate cancers found on patients from the MRI group fulfil criteria for clinically insignificant disease $($ Gleason $\leq 6$, PSA $<10 \mathrm{ng} / \mathrm{ml}$, clinical stage T1/2a) [24].

The risks of TRUS prostate biopsy are well documented with a recent concern over the rising problem of fluoroquinolone resistant infections [25-27] as well as the potential negative psychological impact of cancer investigation and invasive biopsy technique. Thus the routine use of MRI in hematospermia may result in over investigation with biopsy and increased detection of clinically insignificant prostate cancer.

\section{Conclusion}

Although MRI is a useful imaging modality to assess the prostate gland in hematospermia it does not change the outcome of this benign natured presentation in the majority of cases. It can result in increased prostate biopsy with no increase in detection rates of clinically significant cancer. We therefore do not support the routine use of MRI in patients > 40years presenting with hematospermia and it should be used in selected cases only to reduce unnecessary prostate biopsy. 


\section{References}

1 Leary FJ, Aguilo JJ: Clinical significance of hematospermia. Mayo Clin Proc 1974;49: 815-817.

2 Leocadio DE, Stein BS: Hematospermia: etiological and management considerations. Int Urol Nephrol 2009;41:77-83.

3 Han M, Brannigan RE, Antenor JA, Roehl KA, Catalona WJ: Association of hemospermia with prostate cancer. J Urol 2004; 172:2189-2192.

4 Ahmad I, Krishna NS: Hemospermia. J Urol 2007;177:1613-1618.

5 Yagci C, Kupeli S, Tok C, Fitoz S, Baltaci S, Gogus O: Efficacy of transrectal ultrasonography in the evaluation of hematospermia Clin Imaging 2004;28:286-290.

6 Zhao H, Luo J, Wang D, Lu J, Zhong W, Wei J, Chen W: The value of transrectal ultrasound in the diagnosis of hematospermia in a large cohort of patients. J Androl 2012;33: 897-903.

7 Torigian DA, Ramchandani P: Hematospermia: imaging findings. Abdom Imaging 2007;32:29-49.

8 Prando A: Endorectal magnetic resonance imaging in persistent hemospermia. Int Braz J Urol 2008;34:171-179.

9 Albertsen PC, Hanley JA, Fine J: 20-year outcomes following conservative management of clinically localized prostate cancer. JAMA 2005;293:2095-2101.

10 Klotz L: Prostate cancer overdiagnosis and overtreatment. Curr Opin Endocrinol Diabetes Obes 2013;20:204-209.

11 Ramsden AR, Williams J: Haematospermia. Trends Urol Men Health 2011;2:13-16.

$12 \mathrm{Ng} \mathrm{YH}$, Seeley JP, Smith G: Haematospermia as a presenting symptom: outcomes of investigation in 300 men. Surgeon 2013;11:35-38.
13 Numao N, Yoshida S, Komai Y, Ishii C, Kagawa M, Kijima T, Yokoyama M, Ishioka J, Matsuoka Y, Koga F, Saito K, Masuda H, Fujii Y, Kawakami S, Kihara K: Usefulness of pre-biopsy multiparametric magnetic resonance imaging and clinical variables to reduce initial prostate biopsy in men with suspected clinically localized prostate cancer. $\mathrm{J}$ Urol 2013;190:502-508.

14 Tan CH, Wei W, Johnson V, Kundra V: Diffusion-weighted MRI in the detection of prostate cancer: meta-analysis. AJR Am J Roentgenol 2012;199:822-829.

15 Neustein P, Hein PS, Goergen TG: Chronic hemospermia due to müllerian duct cyst: diagnosis by magnetic resonance imaging. $\mathbf{J}$ Urol 1989;142:828.

16 Furuya S, Furuya R, Masumori N, Tsukamoto T, Nagaoka M: Magnetic resonance imaging is accurate to detect bleeding in the seminal vesicles in patients with hemospermia. Urology 2008;72:838-842.

17 Li BJ, Zhang C, Li K, Zhang J, Zhang Y, Sun ZY, Kang HY, Zhou B, Jin FS, Zhang KQ, Li YF: Clinical analysis of the characterization of magnetic resonance imaging in 102 cases of refractory haematospermia. Andrology 2013;1:948-956.

18 Kono M, Kurokawa T, Takata M, Komatsu K, Tsukahara K, Kurose N: Localized amyloidosis of the seminal vesicle: a case report. Hinyokika Kiyo 2011;57:99-101.

19 Foahom Kamwa AD, Mateus C, Thanigasalam R, Boulay-Coletta I, Duchatelle V, Triller M, Robert C, Baumert H: Seminal vesicle metastasis of cutaneous malignant melanoma: an unusual and challenging presentation. Can Urol Assoc J 2015;9:E220 223.
20 Akhter W, Khan F, Chinegwundoh F: Should every patient with hematospermia be investigated? A critical review. Cent European J Urol 2013;66:79-82.

21 Magoha GA, Magoha OB: Aetiology, diagnosis and management of haemospermia: a review. East Afr Med J 2007;84:589-594.

22 Thompson IM, Ankerst DP, Chi C, Goodman PJ, Tangen CM, Lucia M, Feng Z, Parnes HL, Coltman CA Jr: Assessing prostate cancer risk: results from the Prostate Cancer Prevention Trial. J Natl Cancer Inst 2006;98:529_ 534

23 Welch HG, Fisher ES, Gottlieb DJ, Barry MJ: Detection of prostate cancer via biopsy in the Medicare-SEER population during the PSA era. J Natl Cancer Inst 2007;99:1395-1400.

24 O'Donnell H, Parker C: What is low-risk prostate cancer and what is its natural history? World J Urol 2008;26:415-422.

25 Feliciano J, Teper I, Ferrandino M, Macchia R, Blank W, Grunberger I, Colon I: The incidence of fluoroquinolone resistant infections after prostate biopsy--are fluoroquinolones still effective prophylaxis? J Urol 2008;179: 952-955.

26 Williamson DA, Roberts SA, Paterson DL, Sidjabat H, Silvey A, Masters J, Rice M, Freeman JT: Escherichia coli bloodstream infection after transrectal ultrasound-guided prostate biopsy: implications of fluoroquinolone-resistant sequence type 131 as a major causative pathogen. Clin Infect Dis 2012;54:1406-1412.

27 Zaytoun OM, Vargo EH, Rajan R, Berglund $\mathrm{R}$, Gordon S, Jones JS: Emergence of fluoroquinolone-resistant Escherichia coli as cause of postprostate biopsy infection: implications for prophylaxis and treatment. Urology 2011; 77:1035-1041. 